\title{
REVIEWS
}

\section{A Systematic Review and Meta-analysis of the Association Between Vitamin K Antagonist Use and Fracture}

\author{
Wendy Fiordellisi, $M D, M S^{7}$, Katherine White, $M D, M M E^{l}$, and Marin Schweizer, $P h D^{1,2}$ \\ 'Division of General Internal Medicine, University of lowa Hospitals and Clinics, lowa City, IA, USA; ${ }^{2}$ Department of Epidemiology, University of lowa \\ College of Public Health, lowa City, IA, USA.
}

BACKGROUND: Vitamin K antagonist (VKA) anticoagulant use is suspected to increase the risk of bone fracture through inhibition of vitamin K-dependent cofactors of bone formation, an effect not seen with non-vitamin $\mathrm{K}$ antagonist oral anticoagulants (NOACs). The purpose of our systematic review and meta-analysis is to investigate the association between VKA use and fracture.

METHODS: We searched PubMed, EMBASE, and Cochrane Library for studies analyzing fracture in adults using VKAs versus controls. Two authors independently reviewed articles. We assessed for risk of bias using the Newcastle-Ottawa Quality Assessment Scale and the Cochrane Risk of Bias Tool and calculated pooled effects using random effects models.

RESULTS: We included 23 articles (22 observational studies and 1 randomized controlled trial), studying $1,121,582$ subjects. There was no increased odds of fracture in VKA users versus controls (pooled OR 1.01, 95\% CI $0.89,1.14$ ) or in VKA users versus NOAC users (pooled OR 0.95, 95\% CI 0.78, 1.15). Subjects using a VKA for 1 year or longer did not have increased odds of fracture (pooled OR 1.07, 95\% CI 0.90, 1.27). Compared to controls, there was increased odds of fracture in women (pooled OR 1.11, 95\% CI 1.02, 1.21) and older VKA users ( $\geq 65$ ) (pooled OR 1.07, 95\% CI 1.01, 1.14).

DISCUSSION: We found no increase in odds of fracture in VKA users versus controls or NOAC users. There was a small increase in odds of fracture among female and elderly VKA users, which may not be clinically important when accounting for other considerations in choosing an anticoagulant. Our findings suggest that, when anticoagulation is necessary, fracture risk should not be a major consideration in choice of an agent. Future studies directly comparing VKA to NOAC users and studies with longer duration of VKA use may be needed.

KEY WORDS: meta-analysis; osteoporosis; systematic reviews.

J Gen Intern Med 34(2):304-11

DOI: $10.1007 /$ s11606-018-4758-2

(c) Society of General Internal Medicine 2018

Electronic supplementary material The online version of this article (https://doi.org/10.1007/s11606-018-4758-2) contains supplementary material, which is available to authorized users.

Received June 12, 2018

Revised October 4, 2018

Accepted November 16, 2018

Published online December 3, 2018

\section{BACKGROUND}

Warfarin and other vitamin $\mathrm{K}$ antagonists (VKAs) inhibit the formation of vitamin $\mathrm{K}$-dependent clotting factors. However, vitamin $\mathrm{K}$ is also a co-factor in bone formation. ${ }^{1}$ Osteocalcin, the principal non-collagenous protein in bone, is incorporated into bone via vitamin $\mathrm{K}-$ dependent gamma-carboxylation. VKA use decreases bone osteocalcin levels, which decreases bone hardness. ${ }^{2-4}$ Increased blood levels of undercarboxylated osteocalcin are associated with decreased bone mineral quality, ${ }^{5}$ but an association between VKA use and decreased bone mineral density has not been found, ${ }^{6,7}$ suggesting that agents can affect bone structure without affecting bone density.

Whether vitamin $\mathrm{K}$ antagonism leads to an increase in bone fracture risk is controversial, as various studies have yielded differing results. One meta-analysis found an increased risk of fracture associated with VKA use in both cross-sectional and longitudinal studies. However, in sub-analysis of longitudinal studies which matched VKA users to controls, the association became insignificant. Analysis of risk ratios across study designs was not performed, which limited the power of the findings.

In recent years, several non-vitamin $\mathrm{K}$ antagonist oral anticoagulants (NOACs), including the direct thrombin inhibitor dabigatran as well as the direct factor Xa inhibitors (rivaroxaban, apixaban, edoxaban, and betrixaban), have emerged as alternatives to VKAs for various indications. NOACs are not thought to interfere with bone metabolism or increase the risk of bone fracture. Therefore, it is important to clarify the risk of fracture due to VKA use to enable physicians and patients to make appropriate patient-centered clinical decisions when anticoagulation is necessary.

Given the uncertainty about VKA use and fracture risk as well as the availability of alternative anticoagulants, we conducted a systematic literature review and meta-analysis to investigate the association between VKA use and bone fracture. We analyzed the odds of overall fracture between VKA users and controls, including NOAC users. We calculated the odds of various fracture types (hip, vertebral, wrist, and rib) associated with VKA use as well as the association between VKA use and fracture by gender, age, and duration of VKA use. 


\section{METHODS}

We followed guidelines for meta-analysis established by the Preferred Reporting Items for Systematic Reviews and MetaAnalysis (PRISMA) and Meta-Analysis and Systemic Reviews of Observational Studies (MOOSE). ${ }^{8,9}$

\section{SEARCH STRATEGY}

We searched PubMed, EMBASE (Elsevier), and Cochrane Library (Wiley) from inception to December 31, 2017, with the help of an expert librarian using subject headings and keywords such as "vitamin K antagonist," "warfarin," "Coumadin," and all other brand names of warfarin, "fracture," "broken bone," and "skeleton fracture" (online appendix A). We used SCOPUS to review references from selected articles to identify additional relevant studies. We reviewed publications in all languages using Google Translate (https://translate.google.com) for non-English articles.

\section{STUDY SELECTION}

We included observational studies (case control, crosssectional, and prospective and retrospective cohort) and clinical trials analyzing bone fracture in adult patients using VKAs versus controls. A control group was defined as a group not taking VKAs and without an increased risk of fracture. As NOACs are not suspected to increase fracture risk, patients using NOACs were considered an appropriate control. We excluded case reports, systematic reviews, meta-analyses, and review articles. We also excluded studies reporting only the outcomes of bone mineral density scores or osteoporosis but not bone fracture, studies not comparing VKA users to an appropriate control group, and studies reporting duplicate data.

\section{DATA EXTRACTION}

Two authors (WF and KW) independently reviewed studies for inclusion and extracted data with a standardized approach (online appendix B). Differences were resolved by consensus. Abstracted variables included study design, location, dates, sample size, demographics (age, gender), method of ascertainment of VKA exposure, duration of use, measure of compliance (prothrombin time or international normalized ratio (INR)), method of ascertainment of fracture, type of fracture, and crude and adjusted measures of effect, both overall and stratified by fracture type, age, gender, and duration of VKA exposure. Risk of bias was assessed using the NewcastleOttawa Quality Scale for case control studies and cohort studies (online appendix C). ${ }^{10} \mathrm{~A}$ modified version of the Newcastle-Ottawa Quality Scale was used for cross-sectional studies (online appendix C). The Cochrane Risk of Bias Tool was used for clinical trials (online appendix C). ${ }^{11}$

\section{STATISTICAL ANALYSIS}

Our primary outcome was overall odds of fracture in VKA users versus controls. When overall fracture risk was not available, odds of hip fracture or vertebral fracture was used. We preferred adjusted ORs over crude data to control for variables that may have contributed to fracture risk. ORs were used to create pooled ORs using generic inverse weighting in a random effects model. ${ }^{12}$ We stratified overall odds of fracture by methodologic subtype. We calculated overall odds of fracture in VKA users versus all controls and NOAC users. Additional subgroup analysis of VKA users versus controls included assessment of ${ }^{1}$ four types of fracture (hip, vertebral, wrist, and rib), ${ }^{2}$ gender, ${ }^{3}$ age $\geq 65$ years, and ${ }^{4}$ duration of VKA use $\geq 1$ year.

Statistical analyses were performed using Review Manager. ${ }^{12}$ Heterogeneity of studies was analyzed using the Cochrane Q statistic chi-square test ${ }^{13}$ and the $I^{2}$ test, ${ }^{14}$ with higher $I^{2}$ percentages suggesting more heterogeneity. We used Comprehensive Meta-Analysis software V3 to assess publication bias using the modified Egger test. ${ }^{15}$

\section{RESULTS}

The systematic literature review yielded 1897 studies. Two hundred eighty-six duplicate articles were excluded, and 1551 articles were excluded after title and abstract review. Sixty fulltext articles were reviewed, of which 37 were excluded (Fig. 1). Twenty-three studies, with a total of 1,121,582 subjects, were included in our final analyses. ${ }^{16-38}$

The characteristics of included studies are outlined in Table 1. Twenty-two of the 23 studies were observational: 8 prospective cohort studies, ${ }^{22,} 23,27,33,35-387$ retrospective cohort studies, ${ }^{16,18,21,24,25,28,30} 3$ case control studies, ${ }^{19,}, 31$, 32 and 4 cross-sectional studies. ${ }^{17,20,26,29}$ One study was a randomized controlled trial. ${ }^{34}$ Four studies directly compared the risk of fracture in VKA users versus NOAC users. ${ }^{24,} 25,28$, ${ }^{34}$ One study included only females, ${ }^{22}$ and one study included only males. ${ }^{38}$ Eleven studies included only patients $\geq 65$ years old. ${ }^{17,} 19,21,22,27,30,31,33,35,36,38$ Five studies specified a duration of VKA use for $\geq 1$ year for at least part of their study population. $^{20-22,27,32}$ Many of the studies focused on a particular study population, i.e., dialysis patients ${ }^{20,39}$ or patients hospitalized with atrial fibrillation. ${ }^{21}$

There was no difference in odds of fracture between VKA users and controls (pooled OR 1.01, 95\% CI 0.89, 1.14, $I^{2}=$ 74\%) (Fig. 2) or between VKA users and NOAC users (pooled OR $0.95,95 \%$ CI $0.78,1.15, I^{2}=56 \%$ ) (Fig. 3). In most subgroup analyses, we found no difference in odds of fracture with VKA use, including individual fracture type (hip, vertebral, wrist, and rib), male VKA users, and those using VKAs for $\geq 1$ year (Table 2). We did find increased odds of fracture in female VKA users (pooled OR 1.11, 95\% CI 1.02, 1.21, $I^{2}=$ $0 \%$ ) and VKA users aged $\geq 65$ years (pooled OR $1.07,95 \% \mathrm{CI}$ $\left.1.01,1.14, I^{2}=0 \%\right)$. 


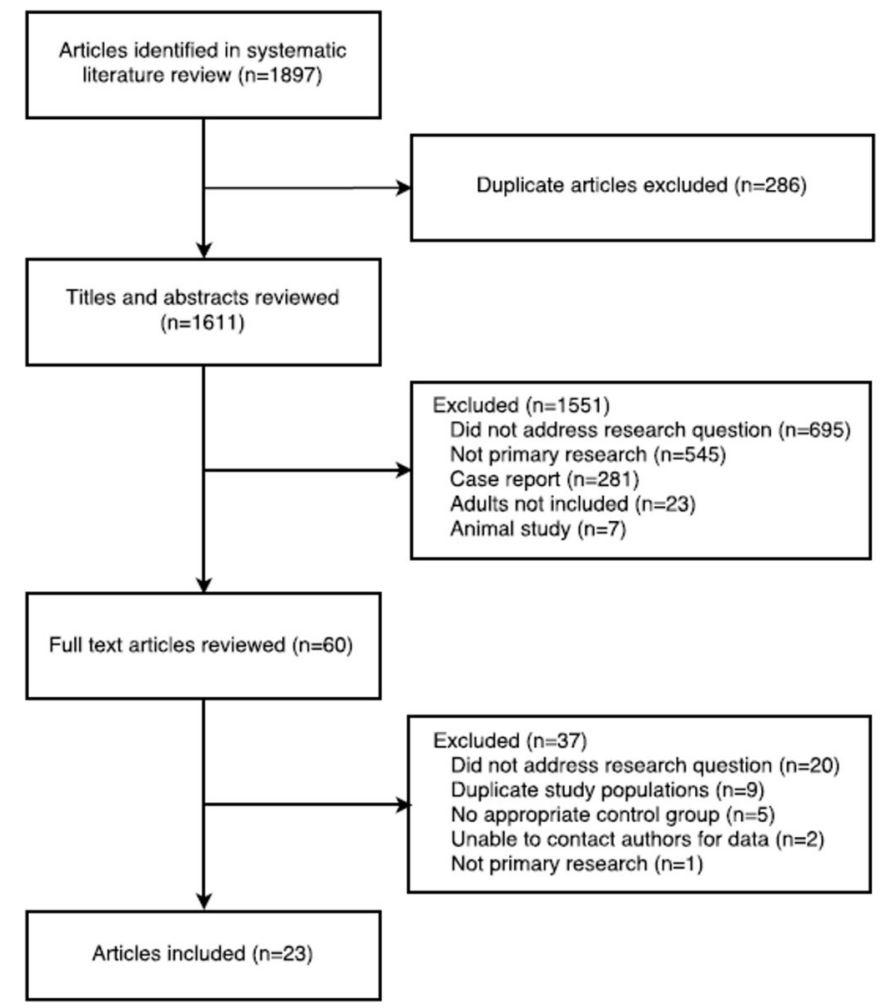

Fig. 1 Study selection diagram.

There was no publication bias by the Egger test (two-tailed $p$ value 0.19 ).

\section{DISCUSSION}

We found that VKA use did not increase the odds of fracture, neither for overall fracture nor for specific types of fracture (hip, vertebral, wrist, or rib). While there were statistically significant increased odds of fracture in female VKA users and VKA users aged 65 years and older versus controls, the clinical significance of these findings is unclear. The odds were small (OR 1.11, 95\% CI 1.02, 1.21 and OR 1.07, 95\% CI 1.01, 1.14, for female VKA users and VKA users aged 65 and older, respectively), and thus likely less important than the other factors that must be considered when choosing an anticoagulant. Such factors include cost, dosing regimen (twice daily dosing required for dabigatran and apixaban), and required laboratory monitoring for VKAs. In addition, the risk of bleeding must be weighed. A meta-analysis from 2015 showed a lower risk of fatal bleeding in NOAC users versus warfarin users (RR 0.53, 95\% CI 0.43, 0.64). ${ }^{40}$ However, reversal agents are currently only available for VKAs and dabigatran. Thus, while the risk of fracture in female VKA users and those aged 65 years and older is statistically significant, the clinical significance seems minimal when accounting for all factors that must be considered in anticoagulant choice.

If exposure to VKAs did inhibit bone formation that resulted in clinically significant bone fracture, we would expect that longer duration of exposure would increase risk. Patients using VKAs for 1 year or longer did not have increased odds of fracture compared to controls. However, the critical duration of VKA exposure that would result in increased risk of fracture is not known. It is possible that the included studies did not evaluate a period of VKA use sufficiently long enough to increase fracture risk. Lai et al. reported the longest duration of VKA use, with a mean duration of use of 3.6 years. ${ }^{23}$ Twelve studies did not specify duration of VKA use. ${ }^{16-18,25 \text {, }}$ 26, 28-30, 35-38

To our knowledge, only one prior meta-analysis studied the risk between VKA use and fracture. Veronese et al. found an increased risk of fracture in VKA users compared to controls in both cross-sectional studies ( 3 studies, pooled RR $1.24,95 \% \mathrm{CI}$ $1.12,1.39$ ) and longitudinal studies (7 studies, pooled RR 1.09, $95 \%$ CI 1.01, 1.18). ${ }^{7}$ However, in analysis of only longitudinal studies comparing VKA users to matched controls, the association became non-significant (2 studies, RR 1.03, 95\% CI 0.90, 1.18). No analysis across study types was reported. Veronese et al. concluded that there was no increased risk of prospectively assessed fracture in VKA users compared to matched medical controls. While we appreciate the rigor of these methods to evaluate risk using high quality data, we would argue that the use of only matched data risks excluding data from studies that are still high in quality. We analyzed data from all studies, then performed sub-analysis of data based on study methodology. We did not find increased odds of fracture overall or in any methodologic design. Using these methods, we included more studies in our analysis than Veronese et al.

We also analyzed four recent studies that directly compared the risk of fracture in VKA users versus NOAC users, 


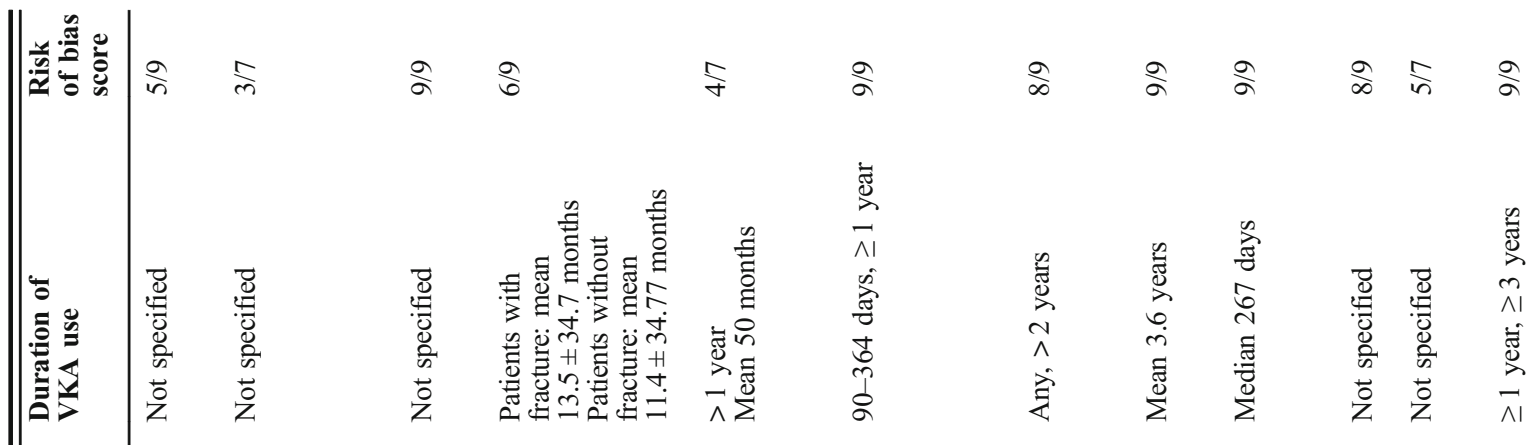

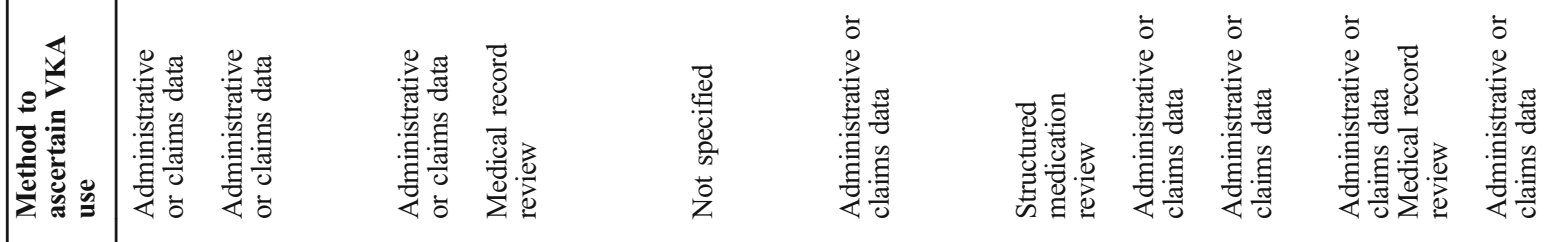

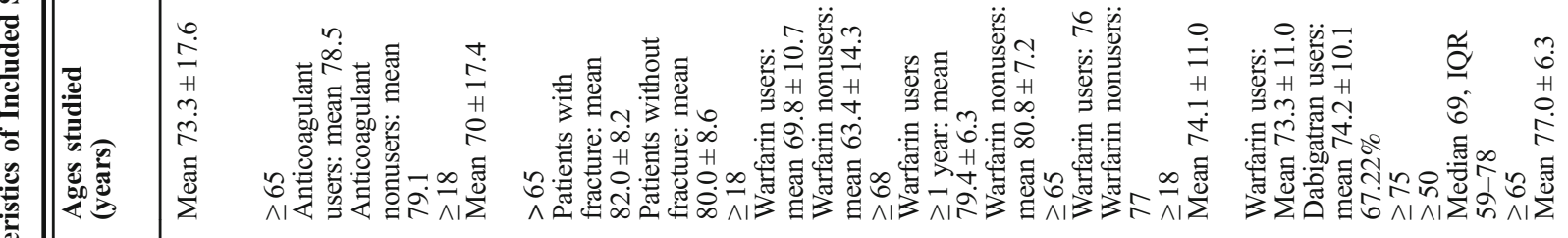

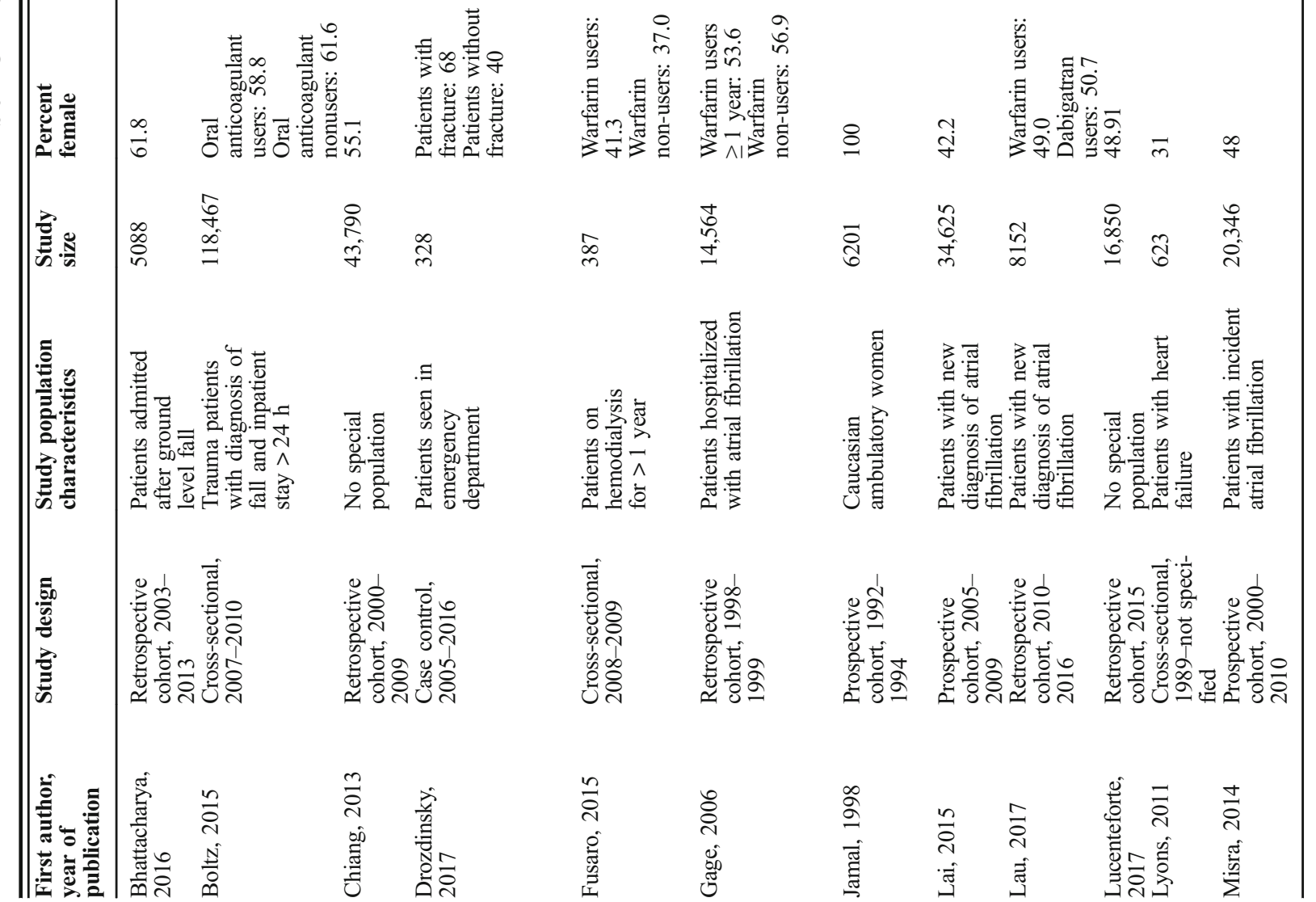




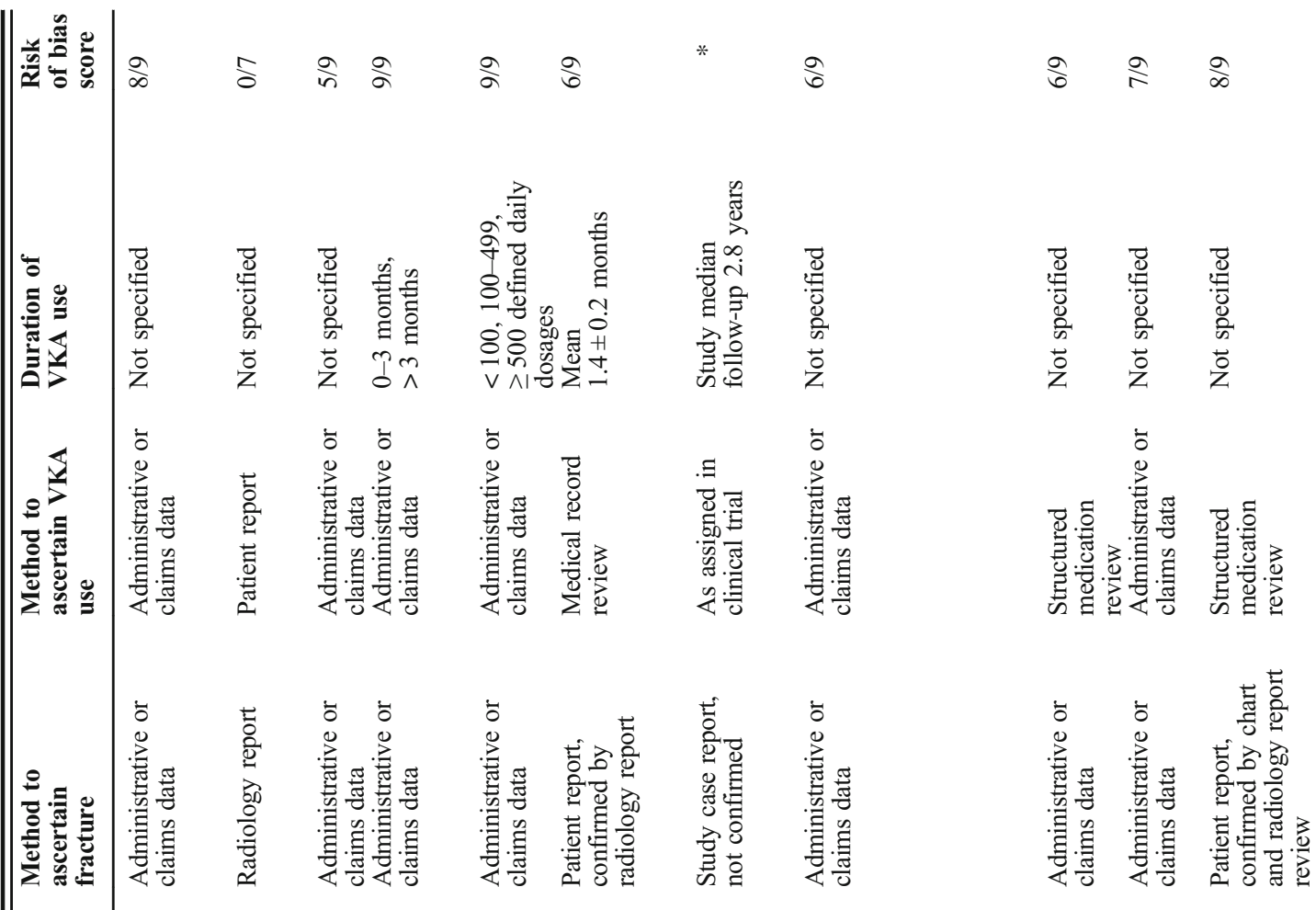
$\ddot{x}$

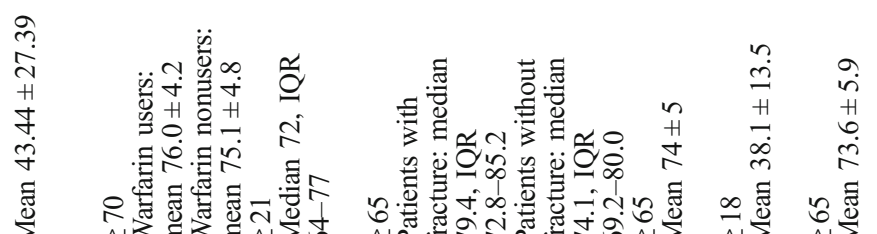

는

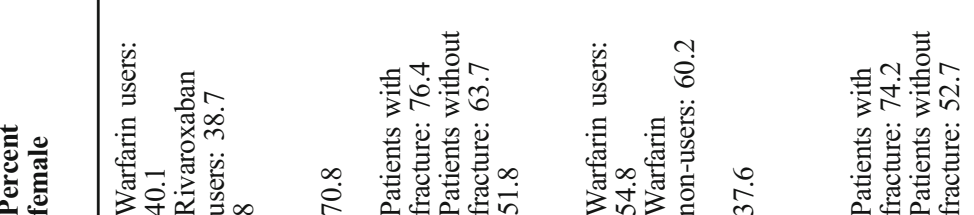

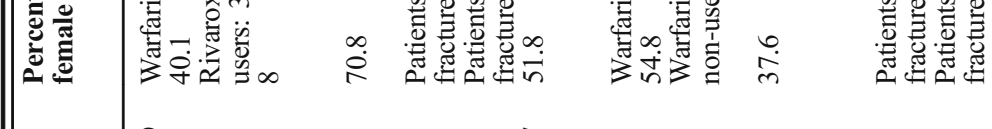

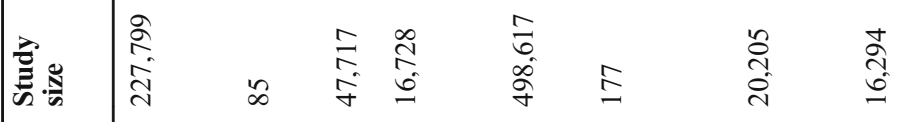

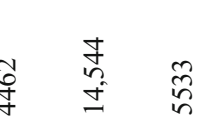

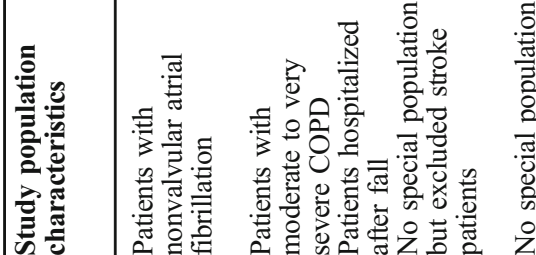

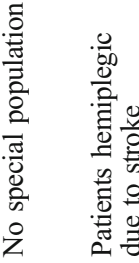

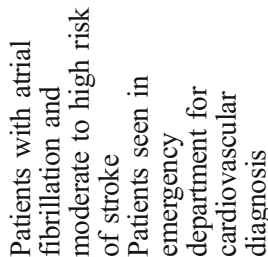

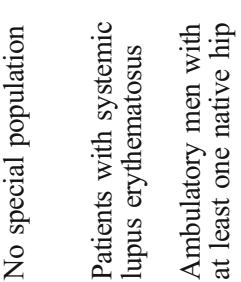

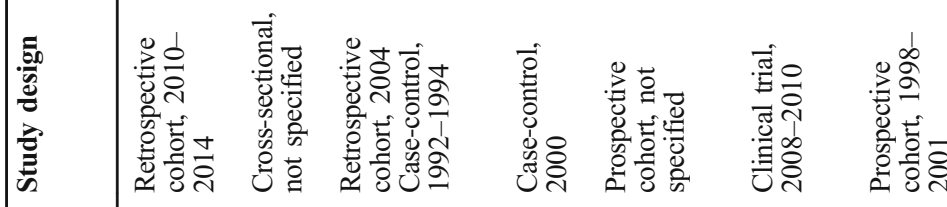

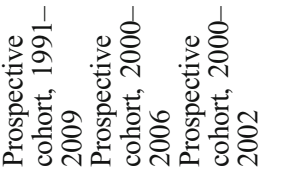

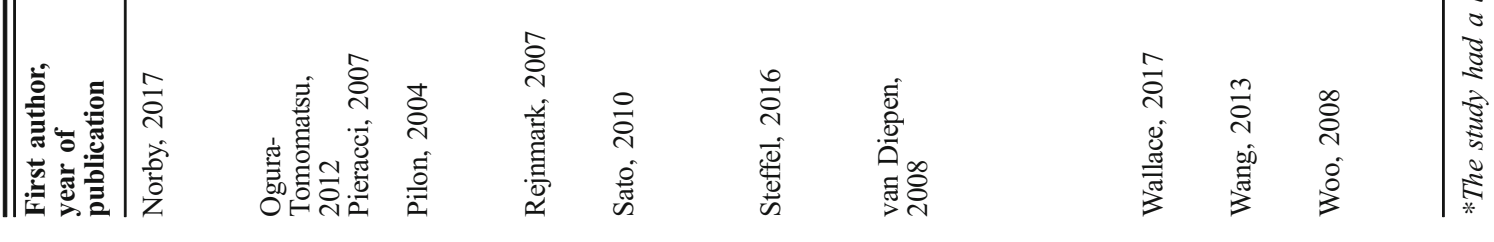




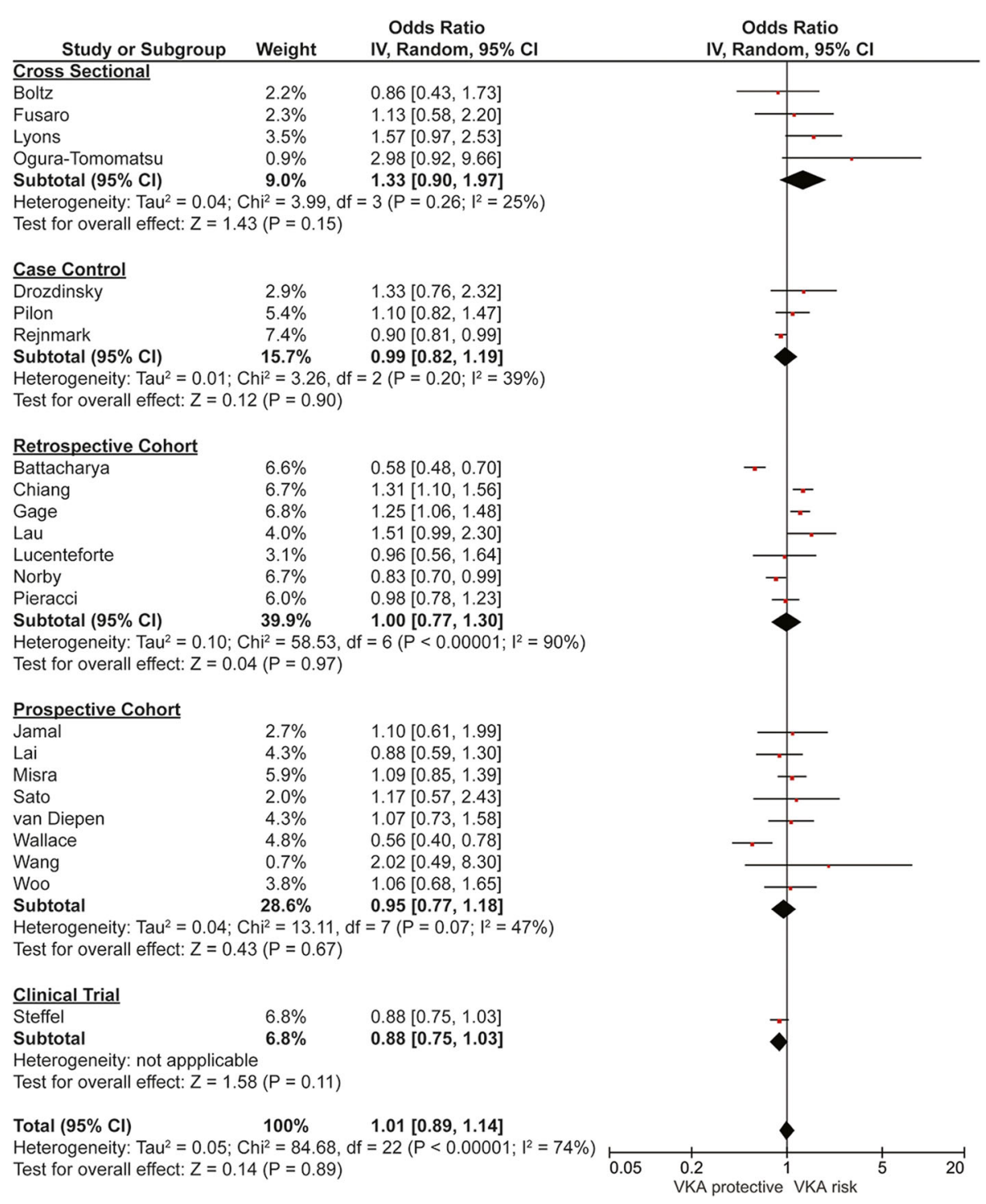

Fig. 2 Pooled odds of fracture in VKA users versus controls, stratified by study design.

including the direct thrombin inhibitor dabigatran ${ }^{24,25}$ and direct factor Xa inhibitors. ${ }^{25,}{ }^{28,}{ }^{34}$ While the mechanisms of action of the direct thrombin inhibitors differ from the direct factor Xa inhibitors, neither are thought to affect bone structure to increase fracture risk. We found no difference in odds of fracture between VKA users and NOAC users.

It has been suspected that VKA use may increase risk of bone fracture by inhibition of vitamin $\mathrm{K}$-dependent gamma- carboxylation of osteocalcin, one of the major proteins in bone that contributes to bone hardness. However, two metaanalyses have shown no association between VKA use and lower bone mineral density. ${ }^{6,7}$ Sugiyama et al. argue that even if bone quantity is decreased by vitamin $\mathrm{K}$ inhibition, an increase in bone quality may compensate. ${ }^{41}$ Thus, reduced bone mineral density may not necessarily increase risk of bone fracture. This might be especially true in weight-bearing sites.

Odds Ratio

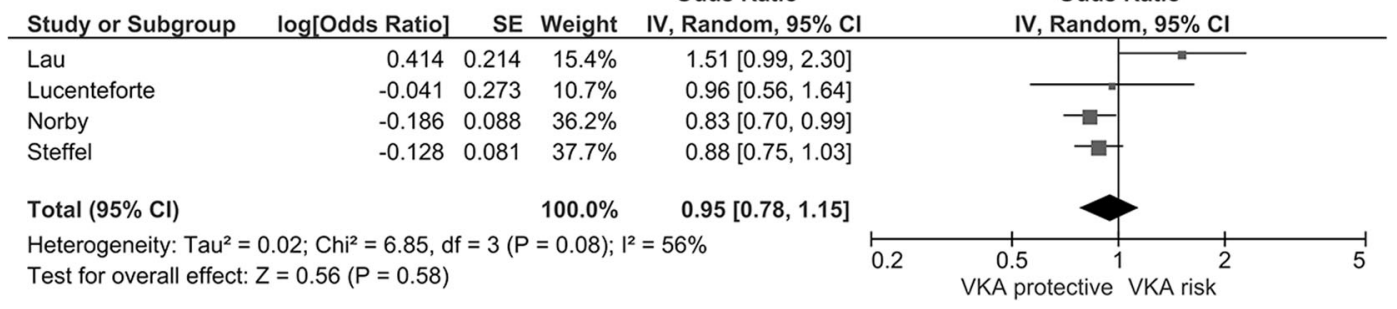

Fig. 3 Pooled odds of fracture in VKA users versus NOAC users. 
Table 2 Sub-analysis of Odds of Fracture in VKA Users Versus Controls

\begin{tabular}{|c|c|c|}
\hline$\overline{\text { Subgroup }}$ & Pooled OR (95\% CI) & Heterogeneity $\left(I^{2}\right)(\%)$ \\
\hline 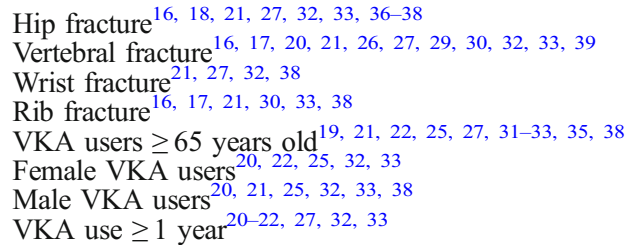 & $\begin{array}{l}0.91(0.69,1.20) \\
1.18(0.96,1.46) \\
1.06(0.87,1.30) \\
1.14(0.86,1.50) \\
1.07(1.01,1.14) \\
1.11(1.02,1.21) \\
1.26(0.93,1.72) \\
1.07(0.90,1.27)\end{array}$ & $\begin{array}{l}85 \\
47 \\
0 \\
55 \\
0 \\
0 \\
67 \\
59\end{array}$ \\
\hline
\end{tabular}

Our meta-analysis did not show increased odds of fracture at weight-bearing sites (hip or spine) or non-weight-bearing sites (wrist or rib).

The quality of our meta-analysis is inherently limited by the quality of the studies included. As 22 of the 23 studies were observational, the strength of the evidence is $2 \mathrm{~B}$ based on GRADE criteria. ${ }^{42}$ There were several sources of potential bias. Selection bias may have played a significant role, as many of the studies examined patients in limited study populations. The patients in these populations may have other risk factors for fracture. For example, dialysis patients are at increased risk of fracture independent of VKA use. ${ }^{42,43}$ In addition, patients of special populations may not represent the general population of VKA users. As most of the included studies are observational, there is risk of misclassification of exposure or outcome. Many studies used administrative or claims data to define VKA use, and most did not confirm patient adherence or maintenance of therapeutic levels. Several studies relied on patient report for ascertainment of fracture. While some studies confirmed patient report with radiologic imaging, some fractures, particularly vertebral and rib fractures, are subclinical and may be missed by patient report.

As NOACs are now widely available, and their mechanism of action is not thought to increase risk of fracture, it is important to clarify the risks of the various anticoagulants so that patients and physicians can make an informed choice of anticoagulant. Four studies directly comparing VKA users to NOAC users revealed no difference in odds of fracture. VKA users did not have increased odds of overall fracture nor specific type of fracture compared to controls, and there was no difference in the odds of fracture in those taking VKAs for 1 year or longer versus controls. While we found increased odds of fracture associated with VKA use in females and patients aged 65 and older, the magnitude was small and likely clinically insignificant when accounting for other factors that must be considered when choosing an anticoagulant. Thus, the results of this meta-analysis suggest that risk of bone fracture should not be a major consideration in anticoagulant choice. Future studies of patients truly representative of VKA users (and not special populations), studies that examine VKA use for longer durations and document compliance, and additional studies that compare VKA use with NOAC use would strengthen this conclusion.
Acknowledgments: We would like to thank Heather Healy for the assistance with the literature search and Robert Wallace and Rich Hoffman for the assistance with editing the manuscript.

Corresponding Author: Wendy Fiordellisi, MD, MS; Division of General Internal MedicineUniversity of Iowa Hospitals and Clinics, Iowa City, IA, USA (e-mail: wendy-fiordellisi@uiowa.edu).

\section{Compliance with Ethical Standards:}

Conflict of Interest: The authors declare that they do not have a conflict of interest.

Publisher's Note Springer Nature remains neutral with regard to jurisdictional claims in published maps and institutional affiliations.

\section{REFERENCES}

1. U.S. Food and Drug Administration [Internet]. Silver Spring, MD: U.S. Food and Drug Administration; c2018 [updated 2015 Oct; cited 2018 Jan 15]. Coumadin Prescribing Information; [about 1 screen]. Available from: http://www.accessdata.fda.gov/drugsatfda_docs/label/2015/ 009218s 115lbl.pdf. Accessed 15 Jan 2018.

2. Kavukcuoglu NB, Patterson-Buckendahl P, Mann AB. Effect of osteocalcin deficiency on the nanomechanics and chemistry of mouse bones. J Mech Behav Biomed Mater. 2009;2(4):348-54.

3. Rey-Sanchez P, Lavado-Garcia JM, Canal-Macias ML, RodriguezDominguez MT, Bote-Mohedano JL, Pedrera-Zamorano JD. Ultrasound bone mass in patients undergoing chronic therapy with oral anticoagulants. J Bone Miner Metab. 2011;29(5):546-51.

4. Sugiyama T, Takaki T, Sakanaka K, Sadamaru H, Mori K, Kato Y, et al. Warfarin-induced impairment of cortical bone material quality and compensatory adaptation of cortical bone structure to mechanical stimuli. J Endocrinol. 2007; 194(1):213-22.

5. Sugiyama T, Kawai $\mathbf{S}$. Carboxylation of osteocalcin may be related to bone quality: a possible mechanism of bone fracture prevention by vitamin K. J Bone Miner Metab. 2001;19(3):146-9.

6. Caraballo PJ, Gabriel SE, Castro MR, Atkinson EJ, Melton Iii LJ. Changes in bone density after exposure to oral anticoagulants: A metaanalysis. Osteoporos Int. 1999;9(5):441-8.

7. Veronese N, Bano G, Bertozzo G, Granziera S, Solmi M, Manzato E, et al. Vitamin $\mathrm{K}$ antagonists' use and fracture risk: results from a systematic review and meta-analysis. J Thromb Haemost. 2015;13(9): 1665-75.

8. Stroup DF, Berlin JA, Morton SC, Olkin I, Williamson GD, Renni D, et al. Meta-analysis of observational studies in epidemiology. JAMA. 2000;283:2008-12.

9. Moher D, Liberati A, Tetzlaff J, Altman DG. Preferred Reporting Items for Systematic Reviews and Meta-Analyses: The PRISMA Statement. J Clin Epidemiol. 2009;62(10):1006-12.

10. The Newcastle-Ottawa Scale (NOS) for assessing the quality of nonrandomised studies in meta-analyses [Internet]. Ottawa: The Ottawa Hospital research Institute; c2018 [updated 2018; cited 2018 Jan 7]. Available from: http://www.ohri.ca/programs/clinical_epidemiology/oxford.asp. Accessed 7 Jan 2018. 
11. Higgins JPT, Altman DG, Gøtzsche PC, Jüni P, Moher D, Oxman AD, et al. The Cochrane Collaboration's tool for assessing risk of bias in randomised trials. BMJ. 2011;343.

12. Review Manager. 5.3 ed. Copenhagen: The Nordic Cochrane Center, The Cochrane Collaboration; 2011.

13. Sheskin D. Handbook of parametric and nonparametric statistical procedures. 5th ed: Boca Raton: Chapman \& Hall/CRC Press; 2011.

14. Higgins JPT, Thompson SG. Quantifying heterogeneity in a metaanalysis. Stat Med. 2002;21(11):1539-58.

15. Harbord RM, Egger M, Sterne JA. A modified test for small-study effects in meta-analyses of controlled trials with binary endpoints. Stat Med. 2006;25(20):3443-57.

16. Bhattacharya B, Maung A, Schuster K, Davis KA. The older they are the harder they fall: Injury patterns and outcomes by age after ground level falls. Injury. 2016;47(9):1955-9.

17. Boltz MM, Podany AB, Hollenbeak CS, Armen SB. Injuries and outcomes associated with traumatic falls in the elderly population on oral anticoagulant therapy. Injury. 2015;46(9):1765-71.

18. Chiang CH, Liu CJ, Chen PJ, Huang CC, Hsu CY, Chen ZY, et al. Hip fracture and risk of acute myocardial infarction: A nationwide study. J Bone Miner Res. 2013;28(2):404-11.

19. Drozdinsky G, Cohen J, Shohat Z, Shiber-Ofer S, Grossman A. Is warfarin usage a risk factor for osteoporotic fractures? A cohort study in the emergency department. Australas Med J. 2017;10(4):329-34.

20. Fusaro M, Tripepi G, Noale M, Plebani M, Zaninotto M, Piccoli A, et al. Prevalence of vertebral fractures, vascular calcifications, and mortality in warfarin treated hemodialysis patients. Curr Vasc Pharmacol. 2015; 13(2):248-58

21. Gage BF, Birman-Deych E, Radford MJ, Nilasena DS, Binder EF. Risk of osteoporotic fracture in elderly patients taking warfarin: results from the National Registry of Atrial Fibrillation 2. Arch Intern Med. 2006; 166(2):241-6.

22. Jamal SA, Browner WS, Bauer DC, Cummings SR. Warfarin use and risk for osteoporosis in elderly women. Study of Osteoporotic Fractures Research Group. Ann Intern Med. 1998;128(10):829-32.

23. Lai HC, Chien WC, Chung $\mathbf{C H}$, Lee WL, Wu TJ, Wang KY, et al. Atrial fibrillation, CHA2DS2-VASc score, antithrombotics and risk of nontraffic-, non-cancer-related bone fractures: A population-based cohort study. Eur J Intern Med. 2015;26(10):798-806.

24. Lau WC, Chan EW, Cheung CL, Sing CW, Man KK, Lip GY, et al. Association Between Dabigatran vs Warfarin and Risk of Osteoporotic Fractures Among Patients With Nonvalvular Atrial Fibrillation. JAMA. 2017;317(11):1151-8

25. Lucenteforte E, Bettiol A, Lombardi N, Mugelli A, Vannacci A. Risk of bone fractures among users of oral anticoagulants: An administrative database cohort study. Eur J Intern Med. 2017;44:e30-e1.

26. Lyons KJ, Majumdar SR, Ezekowitz JA. The unrecognized burden of osteoporosis-related vertebral fractures in patients with heart failure. Circ Heart Fail. 2011;4(4):419-24.

27. Misra D, Zhang Y, Peloquin C, Choi HK, Kiel DP, Neogi T. Incident longterm warfarin use and risk of osteoporotic fractures: propensity-score matched cohort of elders with new onset atrial fibrillation. Osteoporos Int. 2014:25(6):1677-84.
28. Norby FL, Bengtson LGS, Lutsey PL, Chen LY, MacLehose RF, Chamberlain AM, et al. Comparative effectiveness of rivaroxaban versus warfarin or dabigatran for the treatment of patients with non-valvular atrial fibrillation. BMC Cardiovasc Disord. 2017;17(1).

29. Ogura-Tomomatsu H, Asano K, Tomomatsu K, Miyata J, Ohmori N Kodama M, et al. Predictors of osteoporosis and vertebral fractures in patients presenting with moderate-to-severe chronic obstructive lung disease. Copd. 2012;9(4):332-7.

30. Pieracci FM, Eachempati SR, Shou J, Hydo LJ, Barie PS. Use of longterm anticoagulation is associated with traumatic intracranial hemorrhage and subsequent mortality in elderly patients hospitalized after falls: analysis of the New York State Administrative Database. J Trauma. 2007;63(3):519-24.

31. Pilon D, Castilloux AM, Dorais M, LeLorier J. Oral anticoagulants and the risk of osteoporotic fractures among elderly. Pharmacoepidemiol Drug Saf. 2004;13(5):289-94.

32. Rejnmark L, Vestergaard $\mathbf{P}$, Mosekilde $\mathbf{L}$. Fracture risk in users of ora anticoagulants: A nationwide case-control study. Int $\mathrm{J}$ Cardiol. 2007;118(3):338-44.

33. Sato Y, Honda Y, Jun I. Long-term oral anticoagulation therapy and the risk of hip fracture in patients with previous hemispheric infarction and nonrheumatic atrial fibrillation. Cerebrovasc Dis. 2010;29(1):73-8.

34. Steffel J, Giugliano RP, Braunwald E, Murphy SA, Mercuri M, Choi Y, et al. Edoxaban Versus Warfarin in Atrial Fibrillation Patients at Risk of Falling: ENGAGE AF-TIMI 48 Analysis. J Am Coll Cardiol. 2016:68(11):1169-78.

35. van Diepen S, Majumdar SR, Bakal JA, McAlister FA, Ezekowitz JA Heart Failure Is a Risk Factor for Orthopedic Fracture. A PopulationBased Analysis of 16,294 Patients. Circulation. 2008;118(19):1946-52.

36. Wallace ER, Siscovick DS, Sitlani CM, Dublin S, Mitchell P, Robbins JA et al. Incident atrial fibrillation and the risk of fracture in the cardiovascular health study. Osteoporos Int. 2017;28(2):719-25.

37. Wang SH, Chang YS, Liu CJ, Lai CC, Chen WS, Chen TJ, et al. Association of systemic lupus erythematosus with a higher risk of cervical but not trochanteric hip fracture: A nationwide population-based study. Arthritis Care Res. 2013;65(10): 1674-81.

38. Woo C, Chang LL, Ewing SK, Bauer DC. Single-point assessment of warfarin use and risk of osteoporosis in elderly men. J Am Geriatr Soc. 2008;56(7):1171-6.

39. Chai-Adisaksopha C, Hillis C, Isayama T, Lim W, Iorio A, Crowther M. Mortality outcomes in patients receiving direct oral anticoagulants: a systematic review and meta-analysis of randomized controlled trials. J Thromb Haemost. 2015;13(11):2012-20.

40. Sugiyama T, Kugimiya F, Kono S, Kim YT, Oda H. Warfarin use and fracture risk: an evidence-based mechanistic insight. Osteoporos Int. 2015;26(3):1231-2.

41. Guyatt GH, Oxman AD, Kunz R, Falck-Ytter Y, Vist GE, Liberati A et al. Going from evidence to recommendations. BMJ. 2008;336(7652): 1049-51.

42. Beaubrun AC, Kilpatrick RD, Freburger JK, Bradbury BD, Wang L, Brookhart MA. Temporal trends in fracture rates and postdischarge outcomes among hemodialysis patients. J Am Soc Nephrol. 2013;24(9): 1461-9.

43. Jadoul M, Albert JM, Akiba T, Akizawa T, Arab L, Bragg-Gresham JL, et al. Incidence and risk factors for hip or other bone fractures among hemodialysis patients in the Dialysis Outcomes and Practice Patterns Study. Kidney Int. 2006;70(7):1358-66. 